\title{
Selection of a Markov perfect Nash equilibrium in a class of differential games
}

\author{
Javier de Frutos · Guiomar \\ Martín-Herrán
}

Received: date / Accepted: date

\begin{abstract}
This paper revisits the problem of how to select an equilibrium in a differential game in the case of multiplicity of Nash equilibria. Most of the previous applied dynamic games literature has considered pre-play negotiations between players, implicitly or explicitly, with the aim of reaching an agreement on the selection of the pair of strategies. The main objective of this paper is to determine what would be the equilibrium to be played without pre-play communications. We study the linear and nonlinear Markov perfect Nash equilibria for a class of well-known models in the literature if pre-play communications are eliminated. We analyze both symmetric and nonsymmetric strategies. We show that the nonlinear strategies are not always the optimal strategies implemented when pre-play communications are removed. We conclude that in the presence of multiple equilibria and without pre-play communications the
\end{abstract}

\footnotetext{
We are grateful to the editor and two anonymous reviewers for valuable comments and suggestions on an earlier draft of this paper. This research is partially supported by MINECO under projects MTM2013-42538-P, MTM2016-78995-P (AEI) (first author) and ECO201452343-P and ECO2017-82227-P (AEI) (second author), and by Junta de Castilla y León VA024P17 co-financed by FEDER funds (EU).

Javier de Frutos

IMUVA and Dpto. Matemática Aplicada. Facultad de Ciencias. Universidad de Valladolid. Campus Miguel Delibes. Paseo Belén 7. 47011 Valladolid, Spain.

Tel.: +34983184680

Fax: +34983423013

E-mail: frutos@mac.uva.es

orcid.org/0000-0002-5012-6061

Guiomar Martín-Herrán

IMUVA and Dpto. Economía Aplicada. Facultad de Ciencias Económicas y Empresariales. Universidad de Valladolid. Avda. Valle Esgueva, 6. 47011 Valladolid, Spain.

Tel.: +34983423330

Fax: +34983423299

E-mail: guiomar@eco.uva.es

orcid.org/0000-0002-9161-2349
} 
equilibria actually implemented are symmetric piecewise linear Markov perfect Nash equilibria at least for a range of initial values of the state variable.

Keywords Multiple equilibria - differential games - Markovian strategies · nonlinear strategies $\cdot$ international pollution control

\section{Introduction}

This paper revisits the problem of how to select an equilibrium in a differential game when there is an infinite number of equilibria. Most of the previous applied dynamic games literature has considered pre-play negotiations between players, implicitly or explicitly, with the aim of reaching an agreement on the selection of the pair of strategies. The seminal paper by Dockner and Long (1993) is a leading example in this literature. ${ }^{1}$ The main objective of this paper is to determine what would be the equilibrium actually implemented without pre-play communications.

A considerably important issue in the area of dynamic games is the sustainability of cooperative strategies. In the dynamic games literature, it is well-known that the Pareto optimum is difficult to achieve because cooperation can be frustrated by a prisoner's dilemma type of situation. If one player stands to receive a lower payoff in the coordinated solution than what he would get in a noncooperative solution, he will find optimal to deviate from the coordinated solution; he may have an incentive to cheat on the agreement. That is, he may choose a different course of action from that prescribed by the agreement. ${ }^{2}$

Different mechanisms have been proposed in the literature to ensure that the cooperative outcome is sustained by a pair of equilibrium strategies (see, for example, Dockner et al. (1996) and Dockner et al. (2000), Ch. 6 for the use of trigger strategies and De Frutos and Martín-Herrán (2015) and the references therein for the use of incentive equilibrium strategies). ${ }^{3}$ One option often used in the applied dynamic games literature to implement cooperative solutions by means of noncooperative play is exploiting the fact that in some models an infinite number of Markov-perfect Nash equilibria (MPNE) exists. In the case of linear-quadratic games, these equilibria correspond to nonlinear strategies (or "nonsingular", using Rowat's (2007) terminology). The idea is to sustain the cooperative outcome over time, or to approach the cooperative outcome as much as possible by looking for the "nearest" Markov-perfect equilibrium

\footnotetext{
1 Other examples of papers that explicitly consider pre-play negotiations between players are the following (the list is not exhaustive): Piga (2000), Itori and Itaya (2001), Wirl and Feichtinger (2002), Benchekroun and Long (2008), and Kossioris et al. (2008).

2 The problem of the sustainability of the coordinated outcomes over time is already solved when the efficient solution is in itself an equilibrium, as in Chiarella et al. (1984), Rincón-Zapatero et al. (2000), and Martín-Herrán and Rincón-Zapatero (2005).

3 Dockner and Sorger (1996) follow a different approach and construct an infinite number of Nash equilibria in a dynamic game of joint exploitation of a productive asset. They show that the efficient stationary stock can approximately be supported as Nash equilibria in stationary Markovian strategies when the discount rate approaches zero.
} 
among the infinite set of possibilities. Nonlinear strategies have received a great attention in the applied dynamic games literature after the publication of the seminal paper by Tsutsui and Mino (1990). Our paper tries to contribute to this literature.

Tsutsui and Mino (1990) provided a method (different from the guessing method) to construct stationary Markov feedback equilibria for differential games with one state variable and an infinite time horizon. They discussed nonlinear strategies in a linear-quadratic game of duopolistic competition with sticky prices. One of the main purposes of the paper was to examine whether it was possible to construct a more efficient feedback equilibrium (more efficient with respect to the linear feedback equilibrium derived using the guessing method) (see footnote 2 of Tsutsui and Mino's paper). This same purpose has been behind the use of nonlinear strategies in the analysis of a large number of economic and managerial problems. One important question related to the use of nonlinear strategies is that there are many equilibria, in fact an infinite number, depending on which pair of nonlinear strategies is chosen. The lack of a boundary condition in the problem implies that multiple feedback Nash equilibria exist. The multiplicity of equilibria requires some criterion for selecting equilibria. Equilibrium selection is the topic of this paper.

As far as we know, most of the literature on multiple equilibria and nonlinear strategies in linear-quadratic differential games has avoided the problem of selecting equilibria by assuming that the agents will be able to coordinate on the best feedback equilibrium. The choice of the most efficient strategy play is the result of pre-play negotiations or communications. In this paper, as in Dockner and Long (1993) and all subsequent research related to this topic, we consider that pre-play communications and cheap talk are equivalent devices. The selection of a specific equilibrium is determined in a pre-play phase where agents have cheap talks and agree on a specific equilibrium. ${ }^{4}$ The negotiations that lead to an implicit and partial cooperation are not included in the original model and as pointed out by Haurie et al. (2012) (page 274), presumably, if they were included, all the strategies would be different from those obtained for the original criterion. Our main objective in this paper is to analyze which equilibrium would be played if pre-play communications are removed.

If there are not pre-play communications then the multiplicity of equilibria leads to the problem of equilibrium selection. Different criteria for selecting equilibria have been proposed in the literature of dynamic games, see, for example, Başar (1977), Driskill (1997), and Cartigny and Michel (2003). The criterion proposed in these papers removes the multiplicity of equilibria through the definition of some desirable properties. Driskill (1997) avoids the problem

\footnotetext{
4 One exception is Zagonari (1998) where no agreement on the selection of the pair of strategies is required because unilateral initiatives are considered. The paper analyzes a model where two group of countries differ in their preferences for consumption goods as well as in their attention to environmental issues. Another exception is Tasneem et al. (2017) where the empirical relevance of the nonlinear equilibria in a two-player common property resource game is examined. Their results show that nonlinear equilibria cannot be ruled out as irrelevant on behavioral grounds.
} 
of nonuniqueness by considering the equilibrium strategies that result from the game with finite horizon $T$ and letting $T$ tend to infinity. Similarly, for a onedimensional linear-quadratic infinite horizon game Cartigny and Michel (2003) prove that the unique Nash equilibrium of each finite-horizon game converges to a unique Nash equilibrium of the given infinite-horizon game. Furthermore, they prove that the finite-horizon games admit equilibrium strategies that converge to a steady state for all fixed data and this steady state corresponds precisely to the linear strategies of one equilibrium in infinite horizon. For linear-quadratic differential games, Başar (1977) proposes an optimal unique selection of an element of the Nash equilibrium set which exhibits robust behavior by being insensitive to additive random perturbation in the state dynamics, i.e. the unique noncooperative solution of a particular stochastic differential game.

Unlike the papers cited above, in this paper we do not impose any additional property to the equilibrium in order to remove the multiplicity of equilibria. We study the linear and nonlinear Markov perfect Nash equilibria for a class of well-known models in the literature if pre-play communications are eliminated. In Dockner and Long (1993), one of the first works to apply Tsutsui and Mino's method to compute the nonlinear Markov perfect strategies of an international pollution control differential game, the authors highlighted different situations (asymmetric players or incomplete information, for example) which would complicate the process of choosing among Nash equilibria.

The literature that focuses on the comparison of the performance of linear and nonlinear strategies with respect to the Pareto solution could be divided in two major groups. ${ }^{5}$ The papers that establish that nonlinear solutions are Pareto-superior to linear strategies belong to the first group, while those papers showing the opposite result belong to the second group. This technique has been applied to a variety of settings, including industrial organization, environmental economics and public economics. All these papers have considered pre-play negotiations between the players, implicitly or explicitly, with the aim of reaching an agreement on the selection of the pair of strategies. The following is a non-exhaustive list of works which can be classified in the first stream of the literature: Dockner and Long (1993), Feichtinger and Wirl (1993), Wirl (1996), Piga (2000), Ihori and Itaya (2001), Rubio and Casino (2002), Wirl and Feichtinger (2002), Benchekroun and Long (2008), Fujiwara (2008, 2009, 2010), Fujiwara and Matsueda (2009). A list of papers belonging to the second stream of the literature includes: Wirl (1994), Wirl and Dockner (1995), Shibata (2002), Rubio and Casino (2003), Wirl (2007).

In this paper, we analyze a well-known linear-quadratic differential game, for clarity and simplicity in the exposition, although our arguments extend to other more general models, even for games outside the class of linear-quadratic games. In the case of more general models, Markov perfect strategies would

\footnotetext{
5 Most of the works in this literature study different problems formulated as linearquadratic differential games. However, there are some exceptions like Kossioris et al. (2008) that analyze the shallow lake pollution control differential game presenting nonlinear dynamics.
} 
not be computed explicitly, but numerically. In this paper, we study the differential game proposed in Dockner and Long (1993) to analyze a transboundary pollution problem in a symmetric two-player setting.

Following Rowat (2007), we focus on the case where the strategies are required to be defined over the whole state space ${ }^{6}$ and there are an infinite number of equilibria. Therefore, in this paper we exclusively consider globally defined strategies. The optimality conditions established in Rowat (2007) do not imply the existence of a unique Nash equilibria. On the contrary, an infinite number of globally defined Nash equilibria exists. Therefore, our research question of how to choose an equilibrium is still relevant.

Our equilibrium selection mechanism proceeds in two stages. In the first stage, we characterize the stationary Markov perfect Nash equilibria of the differential game using the method proposed in Rincón-Zapatero et al. (1998) (see also Martín-Herrán and Rincón-Zapatero (2002) and Rincón-Zapatero (2004)). This method directly characterizes the stationary strategies candidate to constitute a MPNE of the infinite horizon differential game by means of a quasilinear system of ordinary differential equations (ODEs), instead of the more complicated, fully nonlinear system of Hamilton-Jacobi-Bellman equations. ${ }^{7}$

In the second stage we show that the selection of a MPNE of the differential game is equivalent to the selection of a pair of initial values of the control variables satisfying the system of ODEs. In absence of pre-play communications this leads to a symmetric game in normal form in which each player independently chooses the initial value of his/her own control variable. We call this auxiliary game "the reduced game." The outcome of the reduced game determines the MPNE of the differential game actually played.

The answer to our main research question can be summarized as follows: the nonlinear strategies are not always the optimal strategies to be implemented when pre-play communications are removed. This result is valid even in the case of identical symmetric players. We analyze both symmetric and nonsymmetric strategies. When symmetric strategies are considered, the results are obtained analytically. In the case of nonsymmetric strategies, the results are characterized numerically. If the initial state (the pollution stock) is below a threshold, the nonlinear strategies are not played regardless of the existence of pre-play communications, except if there exists a binding agreement that forces the players to maintain the prior agreement. The equilibrium effectively played is a piecewise linear symmetric MPNE. This equilibrium gives the lowest payoff for both players with symmetric MPNE. If the initial pollution stock is above a threshold, the equilibrium played is a symmetric nonlinear MPNE that gives the closest asymptotic steady-state to the cooperative steady-state.

The paper is organized as follows. In Section 2, we briefly recall the model in Dockner and Long (1993) and present some preliminary results on the characterization of the symmetric and nonsymmetric nonlinear feedback strategies.

\footnotetext{
6 Dockner and Wagener (2014) consider strategies with a local support.

7 See Dockner and Wagener (2014) for a (in this case) mathematically equivalent alternative approach using a shadow price auxiliary system of ordinary differential equations.
} 
Section 3 analyzes what we call the reduced game and collects our main results. Section 4 concludes the paper.

\section{The model and preliminary results}

We consider an infinite time horizon noncooperative two-player differential game. Player i's objective is to maximize

$$
\begin{aligned}
& W_{i}\left(u_{1}, u_{2}, x_{0}\right):=\int_{0}^{\infty} f_{i}\left(x, u_{1}, u_{2}\right) e^{-\rho t} d t, \\
& \text { s.t.: } \dot{x}=g\left(x, u_{1}, u_{2}\right), \quad x(0)=x_{0} .
\end{aligned}
$$

For simplicity in the exposition and with the objective of comparing our results with those obtained in previous literature, we focus on a particular linear-quadratic model that has been extensively studied in the environmental economics literature. The formulation is borrowed from Dockner and Long (1993). The control variables, denoted by $u_{i}, i=1,2$, are the emissions of the two players (countries). A natural assumption is that $u_{i} \geq 0$. The state variable, denoted by $x$, represents the stock of pollution, and is assumed to be positive. Its dynamics is defined by the linear ordinary differential equation:

$$
\dot{x}=g\left(x, u_{1}, u_{2}\right):=u_{1}+u_{2}-\alpha x, \quad x(0)=x_{0},
$$

where parameter $\alpha>0$ denotes the natural absorption rate. The objective of player $i$ is defined by functional (1) with

$$
f_{i}\left(x, u_{i}, u_{j}\right):=u_{i}\left(A-\frac{1}{2} u_{i}\right)-\frac{1}{2} \varphi x^{2},
$$

where $A$ and $\varphi$ are positive parameters.

We are interested in Markovian strategies and, given that the problem is autonomous and the game is played over an infinite time horizon, we restrict ourselves to consider stationary (time-independent) strategies defined over the whole state space. ${ }^{8}$ More precisely we have the following definition,

Definition 1 Let $\mathbb{R}_{+}$denote the set of nonnegative real numbers. The pair $\left(\phi_{1}, \phi_{2}\right)$ of functions $\phi_{i}: \mathbb{R}_{+} \rightarrow \mathbb{R}_{+}, i=1,2$, is a pair of admissible stationary strategies if for all $x_{0} \in \mathbb{R}_{+}$the state equation (3) with $u_{i}(t)=\phi_{i}(x(t))$, $i=1,2$, has a unique absolutely continuous solution $x(t)$ defined for all $t \geq 0$.

We further restrict the set of strategies by observing that given the strategy of player $i$, player $j$ will always choose a strategy $\phi_{j}$ such that $u_{j}(t)=$ $\phi_{j}(x(t)) \leq A$ for almost all $t \geq 0$. The constant choice $u_{i}=A$ corresponds to a myopic player that maximizes its instantaneous utility without taking

8 We focus on globally defined strategies to avoid the discussion about whether or not our results depend on the local or global character of the strategies. 
into account the evolution of the pollution stock over time (Rubio and Casino (2002), see also Proposition 3 in Dockner and Long (1993)). ${ }^{9}$

Proposition 1 Let $\left(\phi_{1}, \phi_{2}\right)$ be a pair of Lipschitz continuous functions defined in $\mathbb{R}_{+}$with $0 \leq \phi_{i}(x) \leq A, i=1,2$, for all $x \in \mathbb{R}_{+}$. Then $\left(\phi_{1}, \phi_{2}\right)$ is a pair of admissible stationary strategies. Furthermore, let $x(\cdot)$ be the solution of (3) with $u_{i}=\phi_{i}(x), i=1,2$, then

$$
e^{-\alpha t} x_{0} \leq x(t) \leq e^{-\alpha t}\left(x_{0}-\frac{2 A}{\alpha}\right)+\frac{2 A}{\alpha}, \quad \forall t \geq 0
$$

and

$$
-\frac{\varphi}{2 \rho} \max \left\{x_{0}^{2}, 4 A^{2} / \alpha^{2}\right\} \leq W_{i}\left(u_{1}, u_{2}, x_{0}\right) \leq \frac{A^{2}}{2 \rho}, \quad i=1,2 .
$$

Proof From the comparison theorem for ordinary differential equations (ODE's) one has that (5) is satisfied for all $t$ in the maximal interval of existence of the unique solution of (3) with $u_{i}=\phi_{i}(x), i=1,2$. Now, using the prolongation theorem for ODE's we conclude that $x(t)$ is defined for all $t$ and $\left(\phi_{1}, \phi_{2}\right)$ is a pair of admissible stationary strategies. The lower and upper bounds in (6) are straightforward from the definition of $W_{i}\left(u_{1}, u_{2}, x_{0}\right)$ in $(1)$, with $f_{i}$ given by (4), observing that from (5) one has $0 \leq x(t) \leq \max \left\{x_{0}, 2 A / \alpha\right\}$.

From now on in this paper, we consider admissible stationary strategies satisfying the conditions in Proposition 1 and we call them simply "admissible stationary strategies." We will use the notation $W_{i}\left(\phi_{1}, \phi_{2}, x_{0}\right)$ to denote $W_{i}\left(u_{1}, u_{2}, x_{0}\right)$ when $u_{i}=\phi_{i}(x)$.

It is well-known that for an infinite horizon dynamic optimization problem there are several non equivalent concepts of optimality (Dockner et al. (2000), Definition 3.2, Haurie et al. (2012), Definition 7.3). However, Proposition 1 shows that, in our case, the infinite integral in (1) with the specification (3)(4) is convergent for all admissible stationary strategies, and therefore, all the definitions of optimality are in fact equivalent in our problem (see Dockner et al. (2000), Haurie et al. (2012)). The following definitions specify the concept of equilibrium relevant for the rest of the paper.

Definition 2 A pair $\left(\phi_{1}, \phi_{2}\right)$ of admissible stationary strategies is a Markov Nash equilibrium (MNE) if for every $x \in \mathbb{R}_{+}$

$$
W_{1}\left(\phi_{1}, \phi_{2}, x\right) \geq W_{1}\left(\psi_{1}, \phi_{2}, x\right), \quad W_{2}\left(\phi_{1}, \phi_{2}, x\right) \geq W_{2}\left(\phi_{1}, \psi_{2}, x\right),
$$

for all $\psi_{1}$ and $\psi_{2}$ such that $\left(\phi_{1}, \psi_{2}\right)$ and $\left(\psi_{1}, \phi_{2}\right)$ are admissible strategies.

\footnotetext{
${ }^{9}$ Rowat (2007) anlyzes the general case without the hypothesis of boundedness of the strategies. The results in that paper show that "a fortiori" the optimal strategies satisfy $u_{j}(t)=\phi_{j}(x(t)) \leq A$ for all $t \geq 0$.
} 
Let us consider the problem in which the objective of player $i$ is to maximize

$$
\begin{aligned}
& W_{i, t}\left(u_{1}, u_{2}, y\right):=\int_{t}^{\infty} f_{i}\left(x, u_{1}, u_{2}\right) e^{-\rho(s-t)} d s, \\
& \text { s.t.: } \dot{x}=g\left(x, u_{1}, u_{2}\right), \quad x(t)=y .
\end{aligned}
$$

We observe that, as problem (1)-(2) is autonomous, every (stationary) MNE for (1)-(2) is also a MNE for (7)-(8) for all $y \in \mathbb{R}_{+}$and all $t \geq 0$. That is, for all $x \in \mathbb{R}_{+}$and $t \geq 0$,

$$
W_{1, t}\left(\phi_{1}, \phi_{2}, x\right) \geq W_{1, t}\left(\psi_{1}, \phi_{2}, x\right), \quad W_{2, t}\left(\phi_{1}, \phi_{2}, x\right) \geq W_{2, t}\left(\phi_{1}, \psi_{2}, x\right) .
$$

An equilibrium with the previous property is called a Markov Perfect Nash Equilibrium (MPNE) (see, for example, Dockner et al. (2000), Definition 4.4). Every stationary MNE is MPNE.

The differential game (7)-(8) is denoted by $\Gamma(t, y)$ using the notation in Dockner et al. (2000) (page 99), being $\Gamma\left(0, x_{0}\right)$ the original differential game (1)-(2).

Definition 3 Let $\left(\phi_{1}, \phi_{2}\right)$ be a stationary MPNE. The value function for player $i, i=1,2$ corresponding to $\left(\phi_{1}, \phi_{2}\right)$ is $V_{i}(x)=W_{i}\left(\phi_{1}, \phi_{2}, x\right), i=1,2$.

The following theorem gives a sufficient condition for a pair of admissible stationary strategies to be a MNE (Dockner et al. (2000), Theorem 4.1) and in consequence a MPNE.

Theorem 1 Let $\left(\phi_{1}, \phi_{2}\right)$ be a set of admissible stationary strategies. Assume that there exist continuously differentiable functions $\left(V_{1}, V_{2}\right)$ defined in $\mathbb{R}_{+}$ satisfying the following system of Hamilton Jacobi-Bellman equations (HJB)

$$
\rho V_{i}(x)=\max _{u \in \mathbb{R}_{+}}\left\{u\left(A-\frac{1}{2} u\right)-\frac{\varphi}{2} x^{2}+\frac{d}{d x} V_{i}(x)\left(u+\phi_{j}-\alpha x\right)\right\}, \quad i \neq j .
$$

Assume that the transversality conditions

$$
\limsup _{t \rightarrow \infty} e^{-\rho t} V_{i}(x(t)) \leq 0, \quad i=1,2,
$$

where $x(\cdot)$ is the solution of (3) with $u_{i}=\phi_{i}(x), i=1,2$, are satisfied for all $x_{0} \in \mathbb{R}_{+}$.

Then if $\phi_{i}(x)$ is a maximizer of the right hand side of (9) for all $x \in \mathbb{R}_{+}$, the pair $\left(\phi_{1}, \phi_{2}\right)$ is a Markov Nash equilibrium.

Proof The proof of the theorem is identical to that of Theorem 4.1 in Dockner et al. (2000) taking into account that, although $V_{i}$ is not bounded below, we can apply Lemma 3.1 in Dockner et al. (2000) because $V_{i}$ is a continuous function and the feasible trajectories are bounded (as previously seen in (5)). 
Remark 1 If $V_{i}, i=1,2$ is continuous and continuously differentiable except at a finite number of points, Theorem 1 is still valid (see Theorem 3.5 in Dockner et al. (2000)).

Note also that (9) is a system of nonlinear ordinary differential equations and that the transversality conditions (10) are not enough to determine a unique solution of the boundary value problem defined by (9) and (10). In particular (10) is satisfied by all stabilising solutions, if they exist. This lack of uniqueness of solutions of (9) and (10) has, as a consequence, the possible existence of an infinite number of MPNE.

For each solution $\left(V_{1}, V_{2}\right)$ of $(9)$, the first-order condition

$$
A-u+\frac{d}{d x} V_{i}(x)=0, \quad i=1,2,
$$

jointly with the nonnegativity condition $u \geq 0$ defines a unique pair of strategies

$$
\phi_{i}(x)=\max \left(0, A+\frac{d}{d x} V_{i}(x)\right), \quad x \in \mathbb{R}_{+}, \quad i=1,2,
$$

that are candidates to constitute a MPNE.

In Rincón-Zapatero et al. (1998) (Section 3) and Martín-Herrán and RincónZapatero (2002) (Theorem 1), it has been proven that, as long as the strategies remain interior, that is $\phi_{i}(x)=A+\frac{d}{d x} V_{i}(x)>0, i=1,2$, the strategies can be computed by means of a system of ordinary differential equations. More precisely, if $\phi_{i}(x)>0$ writing $h_{i}(x)=\phi_{i}(x)$, the pair of functions $\left(h_{1}(x), h_{2}(x)\right)$ satisfies

$$
M\left(h_{1}, h_{2}, x\right)\left[\begin{array}{l}
h_{1}^{\prime} \\
h_{2}^{\prime}
\end{array}\right]=\left[\begin{array}{cc}
\rho+\alpha & 0 \\
0 & \rho+\alpha
\end{array}\right]\left[\begin{array}{c}
h_{1}-A \\
h_{2}-A
\end{array}\right]+\left[\begin{array}{c}
\varphi x \\
\varphi x
\end{array}\right]
$$

where,

$$
M\left(h_{1}, h_{2}, x\right)=\left[\begin{array}{cc}
h_{1}+h_{2}-\alpha x & h_{1}-A \\
h_{2}-A & h_{1}+h_{2}-\alpha x
\end{array}\right] .
$$

Let us remark that given an initial state $x_{0}$, there exists a solution of (12) for each choice of initial values

$$
\left[h_{1}\left(x_{0}\right), h_{2}\left(x_{0}\right)\right]^{T}=\left[\eta_{1}, \eta_{2}\right]^{T} \in \mathcal{H}\left(x_{0}\right),
$$

where, for each $x_{0} \geq 0, \mathcal{H}\left(x_{0}\right)$ is the set of regular points defined by

$$
\mathcal{H}\left(x_{0}\right)=\left\{\left[\eta_{1}, \eta_{2}\right]^{T} \in \mathbb{R}^{2} \mid \operatorname{det} M\left(\eta_{1}, \eta_{2}, x_{0}\right) \neq 0\right\} .
$$

Dockner and Long (1993) and Rowat (2007) (see also Rubio and Casino (2002)), have proved that if condition

$$
\alpha^{2}+3 \alpha \rho+2 \rho^{2}<\varphi
$$

is satisfied, then an infinite number of symmetric globally defined MPNE exists. In this paper, we consider that the model parameters satisfy condition (14). 
Imposing symmetry, $h_{1}(x)=h_{2}(x)=h(x)$, system (12) reduces to the single ordinary differential equation

$$
(3 h-A-\alpha x) h^{\prime}=(\rho+\alpha)(h-A)+\varphi x .
$$

It is worth noting that, although written in a slightly different form, equation (15) coincides with the ordinary differential equation used in Dockner and Long (1993) to derive nonlinear MPNE of the linear quadratic game considered in this paper. If $h_{D L}(x)=h(x)-(A+\alpha x) / 3$, with $h$ a solution of (15), then $h_{D L}$ satisfies the differential equation (C3) in Dockner and Long (1993). On the other hand, our differential game can be transformed in the differential game analyzed in Rowat (2007) changing the objective functional of player $i$ by $2 W_{i}\left(u_{1}, u_{2}, x_{0}\right)-A^{2} / \rho$. Therefore, the conclusions in both papers readily translate to this paper. In what follows we summarize these conclusions.

Equation (15) possesses (Dockner and Long (1993)) two linear solutions defined by

$$
h_{a}(x)=B_{a}+C_{a} x, \quad h_{b}(x)=B_{b}+C_{b} x,
$$

where

$$
B_{j}=\frac{A}{3}-F_{j} \frac{E}{D}, \quad C_{j}=F_{j}+\frac{\alpha}{3}, \quad j=a, b .
$$

Coefficients $F_{j}, D$ and $E$ are given by

$$
\begin{aligned}
D & =\frac{\rho \alpha+\alpha^{2}+3 \varphi}{3}, & E & =\frac{2 A(\rho+\alpha)}{3}, \\
F_{a} & =\frac{\rho+\sqrt{\rho^{2}+12 D}}{6}, & F_{b} & =\frac{\rho-\sqrt{\rho^{2}+12 D}}{6} .
\end{aligned}
$$

Each one of the linear solutions defines two symmetric piecewise linear (affine) MPNE, $\phi_{1}=\phi_{2}=\phi_{b}$ and $\phi_{1}=\phi_{2}=\phi_{a b}$ with

$$
\begin{aligned}
\phi_{b}(x) & =\max \left(h_{b}(x), 0\right), \\
\phi_{a b}(x) & =\max \left(\min \left(h_{a}(x), h_{b}(x)\right), 0\right) .
\end{aligned}
$$

Note that functions $h_{a}$ and $h_{b}$ intersect at point $x^{*}=E / D$, and therefore, $\phi_{a b}(x)=\phi_{b}(x)$ for $x \geq x^{*}$. The piecewise linear strategy $\phi_{a b}$ has been identified in Rowat $(2007)$ where it is proven that the pairs $\left(\phi_{b}, \phi_{b}\right)$ and $\left(\phi_{a b}, \phi_{a b}\right)$ are actually globally defined MPNE.

Let us denote by $h_{c}(x)$ the solution of (15) satisfying $h_{c}(0)=0$ and let us define $\phi_{c}(x)=\max \left(h_{c}(x), 0\right)$. The strategies $\phi_{1}=\phi_{2}=\phi_{c}$ define a lower bound for the set of symmetric nonlinear global MPNE (see below).

For each $x_{0} \in \mathbb{R}_{+}$let us define the set

$$
H\left(x_{0}\right)=\left\{\eta \in \mathbb{R}_{+} \mid \phi_{c}\left(x_{0}\right) \leq \eta \leq \phi_{a b}\left(x_{0}\right)\right\} .
$$

Given an initial state $x_{0} \in \mathbb{R}_{+}$, for each choice of $\eta \in H\left(x_{0}\right)$ there exists a (globally defined) symmetric MPNE $\phi_{1}=\phi_{2}=\max (h(x), 0)$ with $h$ the solution of (15) with $h\left(x_{0}\right)=\eta$. These MPNE correspond to the global MPNE labelled $\hat{\phi}_{3}$ in Theorem 1 of Rowat (2007). 


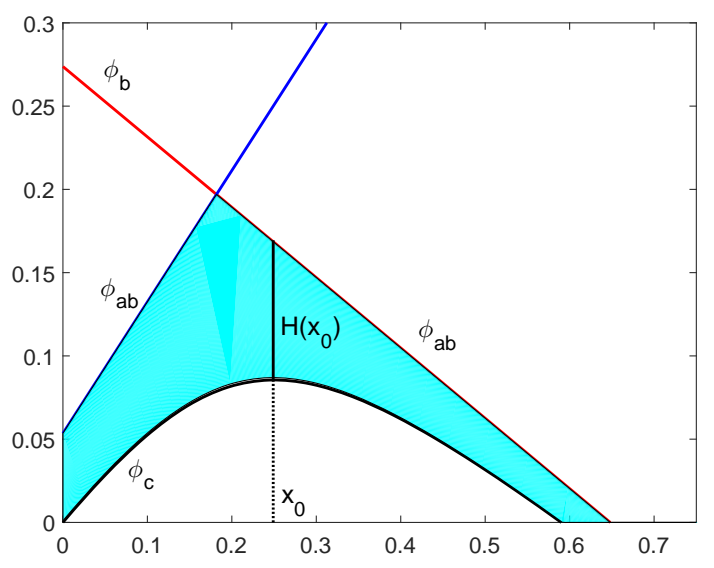

Fig. 1 The set $H\left(x_{0}\right)$ defined in (18) (continuous line in the shaded region). The MPNE $\phi_{b}, \phi_{a b}$ and $\phi_{c}$ for the values of the parameters $A=0.5, \phi=1, \rho=0.1$ and $\alpha=0.5$.

The nonlinear strategies defined by condition $h\left(x_{0}\right)=\eta$ can be explicitly computed (see Dockner and Long (1993), Rubio and Casino (2002), and Rowat (2007)). Outside of region $H\left(x_{0}\right)$ the symmetric nonlinear strategies are locally defined and/or singular. As previously noted, we rule out these strategies and focus exclusively on globally defined MPNE. The set $H\left(x_{0}\right)$ defined in (18) is represented in Figure 1 by the continuous line in the shaded region. In this figure we also represent the piecewise linear MPNE $\phi_{b}$ and $\phi_{a b}$, and the nonlinear MPNE $\phi_{c}$.

We remark that there clearly exist nonsymmetric solutions of (12). It is not the purpose of this paper to carry out an exhaustive analysis of all possible nonsymmetric equilibria of the differential game. Given the main objective of the paper it is enough to restrict the analysis to nonsymmetric equilibria passing through a point in $H\left(x_{0}\right)$. More precisely, for given data $\eta_{1} \in H\left(x_{0}\right)$, $\eta_{2} \in H\left(x_{0}\right)$ with $x_{0} \in \mathbb{R}_{+}$, let $\left(h_{1}(x), h_{2}(x)\right)$ be the solution of (12) with $h_{1}\left(x_{0}\right)=\eta_{1}$ and $h_{2}\left(x_{0}\right)=\eta_{2}$. We assume, without loss of generality, that $\eta_{1} \leq \eta_{2}$. Denoting by $x_{1}^{*}>0$ the point satisfying $h_{1}\left(x_{1}^{*}\right)=0$, assumption $\eta_{1} \leq \eta_{2}$ implies that $h_{2}\left(x_{1}^{*}\right) \geq 0$. Let $\tilde{h}_{2}(x)$ be the solution of the differential equation

$$
\left(\tilde{h}_{2}-\alpha x\right) \tilde{h}_{2}^{\prime}=(\rho+\alpha)\left(\tilde{h}_{2}-A\right)+\varphi x,
$$

with $\tilde{h}_{2}\left(x_{1}^{*}\right)=h_{2}\left(x_{1}^{*}\right)$. Let us denote by $x_{2}^{*}\left(x_{2}^{*} \geq x_{1}^{*}\right)$ the point with $\tilde{h}_{2}\left(x_{2}^{*}\right)=0$. We define

$$
\left(\phi_{1}(x), \phi_{2}(x)\right)= \begin{cases}\left(h_{1}(x), h_{2}(x)\right), & 0 \leq x \leq x_{1}^{*} \\ \left(0, \tilde{h}_{2}(x)\right), & x_{1}^{*} \leq x \leq x_{2}^{*} \\ (0,0), & x \geq x_{2}^{*}\end{cases}
$$

It is possible to show numerically that if $\eta_{i} \in H\left(x_{0}\right), i=1,2$, equation (3) with $u_{i}=\phi_{i}(x)$, defined by (20) has an asymptotically stable positive steady-state, 
so that the transversality condition (10) is satisfied and the pair $\left(\phi_{1}(x), \phi_{2}(x)\right)$ constitutes a nonsymmetric MPNE defined for all $x \in \mathbb{R}_{+}$.

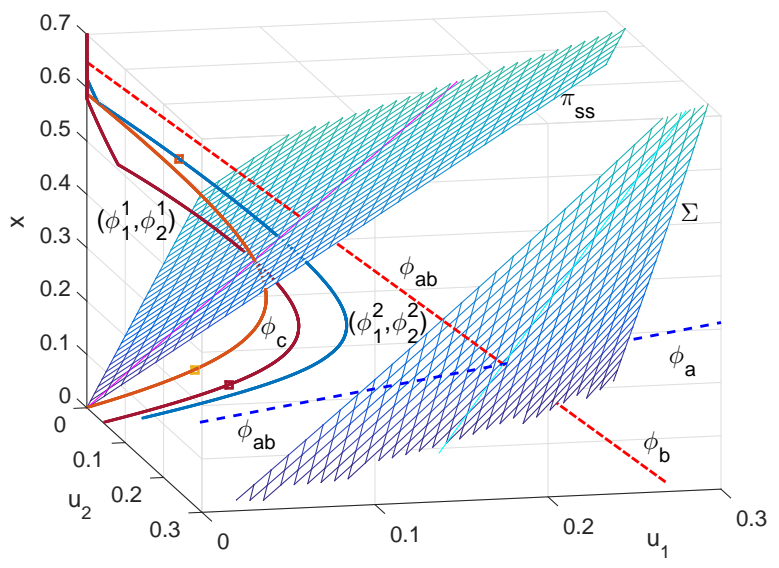

Fig. 2 Nonsymmetric MPNE labelled $\left(\phi_{1}^{r}, \phi_{2}^{r}\right), r=1,2$. The plane of possible steady-states labelled $\Pi_{s s}$ and part of the locus of singular points labelled $\Sigma$.

In Figure 2 we represent two different nonsymmetric MPNE, $\left(\phi_{1}^{1}, \phi_{2}^{1}\right)$ and $\left(\phi_{1}^{2}, \phi_{2}^{2}\right)$, corresponding to the initial values $\left(\phi_{1}^{1}(0.1), \phi_{2}^{1}(0.1)\right)=(0.06,0.1)$ and $\left(\phi_{1}^{2}(0.5), \phi_{2}^{2}(0.5)\right)=(0.04,0.06)$, respectively. For reference we also represent the symmetric MPNE $\phi_{b}, \phi_{a b}$, and $\phi_{c}$. This figure includes the plane $\Pi_{s s}$ of possible stationary steady-states for the dynamics (3) defined by the equation $u_{1}+u_{2}-\alpha x=0$, as well as part of the cone of singular points (labelled $\Sigma$ ) defined by $\operatorname{det} M\left(u_{1}, u_{2}, x\right)=0$ where $M$ is the matrix defined in (13). The possible (symmetric or nonsymmetric) MPNE remain in the interior of $\Sigma$.

For illustration purposes, the left-hand chart in Figure 3 shows the optimal time trajectories of the controls and the right-hand chart shows the optimal time trajectory of the state when players use the strategies $u_{i}=\phi_{i}^{1}(x), i=$ 1,2 , where $\left(\phi_{1}^{1}, \phi_{2}^{1}\right)$ is the MPNE represented in Figure 2 and $x_{0}=0.1$. We recall that $\left(\phi_{1}^{1}, \phi_{2}^{1}\right)$ is univocally determined by conditions $\left(\phi_{1}^{1}(0.1), \phi_{2}^{1}(0.1)\right)=$ $(0.06,0.1)$. More precisely, the right-hand chart in Figure 3 represents $x(t)$, $t \geq 0$, the solution of $(3)$ with $u_{i}=\phi_{i}^{1}(x), i=1,2$ and $x(0)=0.1$. The lefthand chart in Figure 3 presents $u_{1}(t)=\phi_{1}^{1}(x(t))$ the lower (continuous line) curve and $u_{2}(t)=\phi_{2}^{1}(x(t))$ the upper (dashed line) curve.

The differential equation (19) can be obtained taking into account that if $\phi_{1}(x)=0$, then the HJB equation (9) for player 2 becomes

$$
\rho V_{2}(x)=\max _{u_{2} \in \mathbb{R}_{+}}\left\{u_{2}\left(A-\frac{1}{2} u_{2}\right)-\frac{\varphi}{2} x^{2}+\frac{d}{d x} V_{2}(x)\left(u_{2}-\alpha x\right)\right\} .
$$



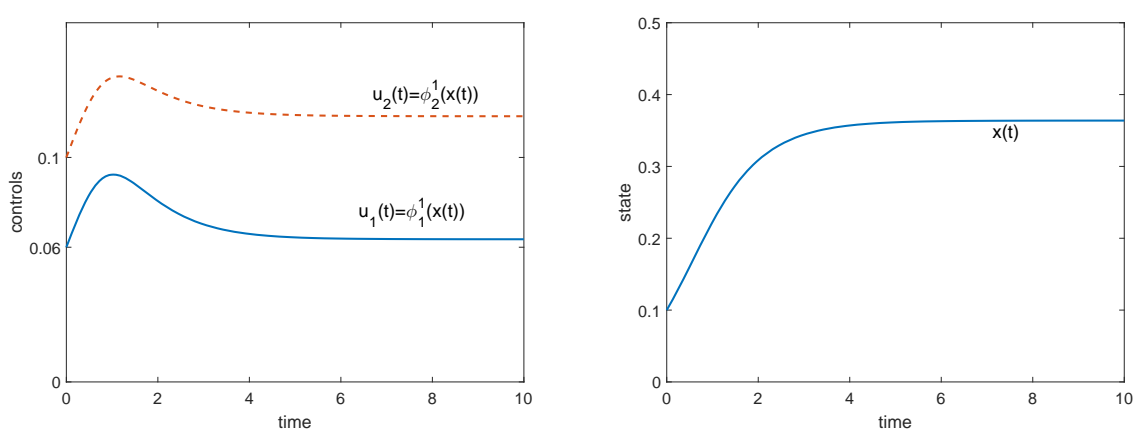

Fig. 3 Optimal time trajectories of the controls (left) and of the state (right) corresponding to the MPNE $\left(\phi_{1}^{1}, \phi_{1}^{2}\right)$ in Figure 2. In the left figure, with continuous line $u_{1}(t)=\phi_{1}^{1}(x(t))$ and with dashed line $u_{2}(t)=\phi_{2}^{1}(x(t))$.

Now using the first-order condition (11) and differentiating the previous equation we get (19) (see Rowat, 2007).

We also remark that, as long as $\phi_{i}>0, i=1,2$, any MPNE should be of the form $\phi_{i}=h_{i}(x), i=1,2$, with $\left(h_{1}(x), h_{2}(x)\right)$ a solution of (12). Although there is no explicit solution of system (12) available, it can be numerically solved without difficulty.

\section{The reduced game}

In view of the analysis of the preceding section it is apparent that, for a given initial state $x_{0}>0$, the players should choose initial conditions for their control variables $\eta_{i} \in H\left(x_{0}\right), i=1,2$ in order to determine which MPNE $\left(\phi_{1}, \phi_{2}\right)$ defined by $(20)$ will be played. We restrict our attention to the region defined by $\eta_{i} \in H\left(x_{0}\right), x_{0}>0$, because it is in this region where we can guarantee the existence of globally defined (not necessarily symmetric) MPNE with $\phi_{i}\left(x_{0}\right)=\eta_{i}$.

We observe that once a choice of initial data $\eta_{i} \in H\left(x_{0}\right), i=1,2$, is made, there is a unique $\operatorname{MPNE}\left(\phi_{1}, \phi_{2}\right)$ such that $\phi_{i}\left(x_{0}\right)=\eta_{i}, i=1,2$. Being a MPNE, the players have no incentive to deviate unilaterally from the feedback strategy $u_{i}=\phi_{i}(x)$. Therefore, the equilibrium $\left(\phi_{1}(x), \phi_{2}(x)\right)$ actually played in the differential game is completely determined by the choice of $\eta_{i}, i=1,2$.

This fact raises the main research question of this paper; that is, which $\eta_{i}$ will player $i$ possibly choose in absence of pre-play communication?

In order to be more precise, from now on the MPNE given by (20) when the initial choice of controls is $\phi_{i}\left(x_{0}\right)=\eta_{i} \in H\left(x_{0}\right), i=1,2$ is denoted by $\left(\phi_{1}\left(x, \eta_{1}, \eta_{2}\right), \phi_{2}\left(x, \eta_{1}, \eta_{2}\right)\right)$.

We can reformulate the problem of choosing $\left(\eta_{1}, \eta_{2}\right)$, given $x_{0}>0$, as a symmetric game in normal form (which we call the reduced game). The set of strategies of the two players is $H\left(x_{0}\right)$, and the payoff functions are defined as 
$V_{i}\left(x_{0}, \cdot, \cdot\right): H\left(x_{0}\right) \times H\left(x_{0}\right) \rightarrow \mathbb{R}$ with $V_{i}\left(x_{0}, \eta_{1}, \eta_{2}\right)$ the value function of player $i, i=1,2$ when the players follow the strategy $u_{i}=\phi_{i}\left(x, \eta_{1}, \eta_{2}\right), i=1,2$, and the initial state is $x(0)=x_{0}$. The reduced game is played in pure strategies.

From now on, we assume that the selection of the equilibrium to be played is the result of the reduced game.

We have the following results

Proposition 2 Let $x_{0}>0$. If $\eta_{1} \leq \eta_{2}, \eta_{i} \in H\left(x_{0}\right), i=1,2$, then

$$
V_{i}\left(x_{0}, \eta_{1}, \eta_{1}\right) \geq V_{i}\left(x_{0}, \eta_{2}, \eta_{2}\right), \quad i=1,2
$$

Proof The symmetry of the game implies $V_{1}\left(x_{0}, \eta, \eta\right)=V_{2}\left(x_{0}, \eta, \eta\right)$ for all $\eta \in H\left(x_{0}\right)$. Therefore, it is enough to prove the proposition for player 1 . Let $(\phi(x, \eta, \eta), \phi(x, \eta, \eta))$ be the (symmetric) solution of (12) with $\phi\left(x_{0}, \eta, \eta\right)=\eta$.

For $\eta \in H\left(x_{0}\right)$, the strategies $u_{1}=u_{2}=\phi(x, \eta, \eta)$ are positive along the solution of (3) with $u_{1}=u_{2}=\phi(x, \eta, \eta)$. Then, using the first-order condition (11) in the HJB equations (9), one has

$$
V_{i}\left(x_{0}, \eta_{i}, \eta_{i}\right)=\frac{1}{\rho}\left(\eta_{i}\left(A-\frac{\eta_{i}}{2}\right)-\frac{\varphi}{2} x_{0}^{2}+\left(\eta_{i}-A\right)\left(2 \eta_{i}-\alpha x_{0}\right)\right), \quad i=1,2 .
$$

Then,

$$
V_{1}\left(x_{0}, \eta_{1}, \eta_{1}\right)-V_{1}\left(x_{0}, \eta_{2}, \eta_{2}\right)=\frac{1}{\rho}\left(\eta_{1}-\eta_{2}\right)\left(\frac{3}{2}\left(\eta_{1}+\eta_{2}\right)-\left(A+\alpha x_{0}\right)\right) .
$$

Taking into account that for $\eta_{1}, \eta_{2} \in H\left(x_{0}\right)$ the inequality

$$
\frac{3}{2}\left(\eta_{1}+\eta_{2}\right)-\left(A+\alpha x_{0}\right) \leq 0
$$

is satisfied, we conclude that $V_{1}\left(x_{0}, \eta_{1}, \eta_{1}\right)-V_{1}\left(x_{0}, \eta_{2}, \eta_{2}\right) \geq 0$.

Remark 2 Proposition 2 shows that if the players restrict themselves to play symmetric strategies, then the MPNE leading to the greatest payoff requires choosing the lowest initial value of the control variable, that is, strategy $\phi_{c}$. This result is consistent with the findings in Dockner and Long (1993), ${ }^{10}$ as well as in their follow-up papers (see, for example, Rubio and Casino (2002)), where the players agree to implement the "best" nonlinear MPNE. Note that this requires previous communication (cheap talk) between the two players in order to agree on the initial data to be chosen. This is the most common point of view in the literature when cheap talk is used to implement the best or most efficient MPNE, which in this case is the equilibrium which gives a steady-state pollution stock as close as possible to the cooperative steady-state pollution stock. However, as next propositions show, if cheap talk is not allowed and depending on the initial value of the state, the outcome could be different.

The following proposition compares the payoffs for nonsymmetric MPNE.

10 Dockner and Long (1993) consider locally defined nonlinear strategies, whereas in this paper we only consider globally defined nonlinear strategies. 
Proposition 3 Let $x_{0}>0$. If $\eta_{1} \leq \eta_{2}, \eta_{i} \in H\left(x_{0}\right)$ and $\left(\eta_{1}+\eta_{2}\right) / 2 \geq-A+$ $\alpha x_{0}$, then

$$
V_{1}\left(x_{0}, \eta_{1}, \eta_{2}\right) \leq V_{2}\left(x_{0}, \eta_{1}, \eta_{2}\right)
$$

Proof For $\eta_{i} \in H\left(x_{0}\right), i=1,2$, the strategies $u_{i}=\phi_{i}\left(x, \eta_{1}, \eta_{2}\right)$ are positive along the solution of (3) with $u_{i}=\phi_{i}\left(x, \eta_{1}, \eta_{2}\right)$. Then, using the first-order condition (11) in the HJB equations (9) one has

$$
V_{i}\left(x_{0}, \eta_{1}, \eta_{2}\right)=\frac{1}{\rho}\left(\eta_{i}\left(A-\frac{\eta_{i}}{2}\right)-\frac{\varphi}{2} x_{0}^{2}+\left(\eta_{i}-A\right)\left(\eta_{1}+\eta_{2}-\alpha x_{0}\right)\right), \quad i=1,2 .
$$

The expressions for the value functions can be easily manipulated to arrive to

$$
V_{2}\left(x_{0}, \eta_{1}, \eta_{2}\right)-V_{1}\left(x_{0}, \eta_{1}, \eta_{2}\right)=\frac{1}{\rho}\left(\frac{\eta_{1}+\eta_{2}}{2}+A-\alpha x_{0}\right)\left(\eta_{2}-\eta_{1}\right) .
$$

Therefore, if $\left(\eta_{1}+\eta_{2}\right) / 2 \geq-A+\alpha x_{0}$ and $\eta_{1} \leq \eta_{2}$, inequality (22) is proven.

Remark 3 Condition $\left(\eta_{1}+\eta_{2}\right) / 2 \geq-A+\alpha x_{0}$ is satisfied for all $\eta_{1}, \eta_{2} \in H\left(x_{0}\right)$ if the model parameters satisfy the following two conditions:

$$
-3+\alpha^{2}+\alpha \rho<0, \quad-3+\alpha^{4}+\left(6-\rho^{2}\right) \alpha^{2}+4 \alpha \rho<0
$$

The first condition is satisfied if $\alpha<1$ and $\rho<1$. The second condition is satisfied if $\alpha$ is moderately small (more precisely if $\alpha<0.47$ ). These two conditions guarantee that the point $x_{b}$ where $\phi_{b}\left(x_{b}\right)=\phi_{a b}\left(x_{b}\right)=0$ satisfies $x_{b}=-B_{b} / C_{b} \leq A / \alpha$. Therefore, if $x_{0} \leq x_{b}$, then $\eta \geq-A+\alpha x_{0}$ for all $\eta \in H\left(x_{0}\right)$, and as a consequence the condition on the mean of $\eta_{1}$ and $\eta_{2}$ in the statement of the proposition is fulfilled. If $x_{0}>x_{b}$ the only choice leading to a MPNE is $\eta_{1}=\eta_{2}=0$.

Remark 4 Proposition 3 shows that the player who gets the highest payoff is the player who initially chooses the highest control variable $(\eta)$. From Proposition 3 we infer that, in absence of pre-play communication, player $i, i=1,2$ has an incentive to choose the MPNE defined with highest possible value of $\eta_{i}$, regardless of the choice of his/her opponent. Note that, due to the symmetry of the game, if $\eta_{1} \leq \eta_{2}$, then $V_{1}\left(x_{0}, \eta_{1}, \eta_{2}\right) \leq V_{2}\left(x_{0}, \eta_{1}, \eta_{2}\right)=V_{1}\left(x_{0}, \eta_{2}, \eta_{1}\right)$ and similarly for player 2 . That is, each player is better off if he or she chooses an initial $\eta \in H\left(x_{0}\right)$ bigger than the choice of his/her opponent.

Note that the pairs of initial emission rates $\left(\eta_{1}, \eta_{2}\right)$ and $\left(\eta_{2}, \eta_{1}\right)$ lead to the same trajectory of the pollution stock. Then, if the environmental problem is not initially extremely important, specifically if $x_{0} \leq x_{b}$, each country would prefer initially to emit at a rate greater than that of its neighbor. This behavior corresponds to a free-riding problem in the sense that the greater the emission rate, the greater the payoff given the fixed trajectory of the pollution stock. Proposition 2 also shows that one country cannot unilaterally reduce the stock of pollution. If the country would reduce its emissions, the pollution stock would decline. The neighbor country benefits from this decline in pollution and 
could raise its emission in order to increase its welfare without increasing the pollution stock, and therefore, without worsening the environmental problem. However, if the environmental problem is initially extremely serious $\left(x_{0}>x_{b}\right)$, then both countries would choose initially null emissions.

The following proposition compares the payoffs for nonsymmetric MPNE in more detail.

Proposition 4 Let $x_{0}>0$ and $\eta_{1} \in H\left(x_{0}\right)$. If $\eta_{2,1} \leq \eta_{2,2}$ with $\eta_{2,1}, \eta_{2,2} \in$ $H\left(x_{0}\right)$, then

$$
V_{2}\left(x_{0}, \eta_{1}, \eta_{2,1}\right) \leq V_{2}\left(x_{0}, \eta_{1}, \eta_{2,2}\right)
$$

if and only if

$$
\left(\eta_{2,1}+\eta_{2,2}\right) / 2+\eta_{1}-\alpha x_{0} \geq 0 .
$$

Proof Reasoning as in Proposition 3 we arrive to

$V_{2}\left(x_{0}, \eta_{1}, \eta_{2,1}\right)-V_{2}\left(x_{0}, \eta_{1}, \eta_{2,2}\right)=\frac{1}{\rho}\left(\eta_{2,1}-\eta_{2,2}\right)\left(\frac{1}{2}\left(\eta_{2,1}+\eta_{2,2}\right)+\eta_{1}-\alpha x_{0}\right)$.

Therefore, if $\eta_{2,1} \leq \eta_{2,2}$, then $V_{2}\left(x_{0}, \eta_{1}, \eta_{2,1}\right)-V_{2}\left(x_{0}, \eta_{1}, \eta_{2,2}\right) \leq 0$ if and only if $\left(\eta_{2,1}+\eta_{2,2}\right) / 2+\eta_{1}-\alpha x_{0} \geq 0$.

Remark 5 Condition (24) is satisfied if both players choose initial data $\eta_{1}, \eta_{2,1}$, $\eta_{2,2}$ above the plane $\Pi_{s s}$ of possible steady states for the dynamics (3). Plane $\Pi_{s s}$ is defined by the equation $u_{1}+u_{2}-\alpha x=0$. If $\left(\phi_{1}(x), \phi_{2}(x)\right)$ is the MPNE played, then the steady state $x_{s s}$ is characterized by $\phi_{1}\left(x_{s s}\right)+\phi_{2}\left(x_{s s}\right)-\alpha x_{s s}=$ 0 .

Note that when condition (24) applies and all the initial choices of the emission rates are above plane $\Pi_{s s}$, then the initial pollution stock is lower than its long-run value, and hence, the pollution stock is increasing from $x_{0}$ to $x_{s s}$ as time goes by.

Remark 6 If condition (24) is satisfied, Proposition 4 establishes that, regardless of the choice of the opponent, each player gets a greater payoff the greater his/her choice of the initial control variable. It is clear that if the equilibrium to be played is chosen as a result of the reduced game, the outcome of the game is both players choosing $\eta_{1}=\eta_{2}=\phi_{a b}\left(x_{0}\right)$. That is, the equilibrium played in the original differential game is the symmetric piecewise MPNE $\phi_{a b}$. In fact if condition (24) is satisfied, then $\eta_{1}=\eta_{2}=\phi_{a b}\left(x_{0}\right)$ is the Nash equilibrium of the reduced game as Proposition 5 shows.

Note that the fulfillment of condition (24) describes whether the countries evaluate the environmental problem as initially serious or not. Proposition 4 shows that if the environmental problem is moderate, each country would prefer to emit at the highest possible rate in order to increase its payoff irrespective of the initial emission rate of its neighbor. However, if the environmental problem is serious, each country would prefer to emit at the lowest possible rate regardless of the initial emission rate selected by its neighbor. 
Proposition 5 Let $\tilde{x}_{0}$ and $\tilde{x}_{1}$ such that $\phi_{c}\left(\tilde{x}_{0}\right)=(\alpha / 2) \tilde{x}_{0}$ and $\phi_{a b}\left(\tilde{x}_{1}\right)=$ $(\alpha / 2) \tilde{x}_{1}$. Then,

1. If $x_{0} \leq \tilde{x}_{0}$, then $\eta_{1}=\eta_{2}=\phi_{a b}\left(x_{0}\right)$ is the only Nash equilibrium of the reduced game.

2. If $x_{0}>\tilde{x}_{1}$, then $\eta_{1}=\eta_{2}=\phi_{c}\left(x_{0}\right)$ is the only Nash equilibrium of the reduced game.

Proof If $x_{0} \leq \tilde{x}_{0}$, then condition (24) is satisfied for all $\eta_{1}, \eta_{2,1}, \eta_{2,2} \in H\left(x_{0}\right)$. Proposition 4 can be rephrased by saying that $\eta_{2,2}\left(\eta_{2,2}>\eta_{2,1}\right)$ is a dominant strategy for player 2 regardless of the strategy $\eta_{1}$ chosen by player 1 . Using the symmetry of the game, if $\eta_{1,1}, \eta_{1,2}, \eta_{2} \in H\left(x_{0}\right)$ with $\eta_{1,1} \leq \eta_{1,2}$, then

$$
V_{1}\left(\eta_{1,1}, \eta_{2}\right) \leq V_{1}\left(\eta_{1,2}, \eta_{2}\right), \quad \forall \eta_{2} \in H\left(x_{0}\right)
$$

Therefore, $\eta_{1,2}\left(\eta_{1,2}>\eta_{1,1}\right)$ is also a dominant strategy for player 1 for all possible choices $\eta_{2}$ of player 2 . Given that $\phi_{a b}\left(x_{0}\right)=\max H\left(x_{0}\right), \eta_{1}=\eta_{2}=$ $\phi_{a b}\left(x_{0}\right)$ constitutes the only Nash equilibrium of the reduced game.

If $x_{0}>\tilde{x}_{1}$, then $\left(\eta_{2,1}+\eta_{2,2}\right) / 2+\eta_{1}-\alpha x_{0} \leq 0$ and consequently from Proposition $4, V_{2}\left(x_{0}, \eta_{1}, \eta_{2,1}\right)-V_{2}\left(x_{0}, \eta_{1}, \eta_{2,2}\right) \geq 0$. Now the dominant strategy for player 2 is $\eta_{2,1}\left(\eta_{2,1}<\eta_{2,2}\right)$ regardless of the choice $\eta_{1}$ of player 1 . The conclusion is reached using the same reasoning as before.

Remark 7 The consequence of Proposition 5 is striking. Even if there is preplay communication and an agreement to implement $\eta_{1}=\eta_{2}=\phi_{c}\left(x_{0}\right)$ has been reached (which gives the highest payoff for both players with symmetric $\mathrm{MPNE}$ ), if $x_{0} \leq \tilde{x}_{0}$ the players have an incentive to deviate from the initial agreement in a sort of prisoner's dilemma. Both players have an incentive to choose a higher initial value of the control variable, and in consequence, the implemented equilibrium is the symmetric piecewise linear MPNE $\left(\phi_{a b}(x), \phi_{a b}(x)\right)$ (which gives the lowest payoff for both players with symmetric MPNE).

If the objective of pre-play communications is to implement the MPNE which gives an asymptotic steady-state as close as possible to the cooperative steady-state (the best MPNE), from Proposition 5 we infer that the objective can only be reached for $x_{0} \geq \tilde{x}_{1}$. In other words, the objective is attainable exclusively if the environmental problem is initially important. Note that this result is consistent with the findings in Rubio and Casino (2002). If this is the case, one can remove pre-play communications and still have the desired outcome. However, if $x_{0} \leq \tilde{x}_{0}$, the objective is unattainable, except if there exists a binding agreement that forces the players to maintain the prior agreement.

Remark 8 It is worth noting that our equilibrium selection mechanism (the outcome of the reduced game) depends on the initial state $x_{0}$. This selection mechanism determines which one of the two MPNE, $\left(\phi_{a b}, \phi_{a b}\right)$ or $\left(\phi_{c}, \phi_{c}\right)$, is effectively implemented by the players. The dependency of the implemented equilibrium on the initial condition is common to other equilibrium selection 
mechanisms, as for example, pre-play communications (see Rubio and Casino (2002)) and is caused by the existence of an infinite number of equilibria.

We would like to emphasize that the selection of the MPNE to be played depends on the initial state $x_{0}$, but the equilibrium does not. Let us denote by $\phi$ either $\phi_{a b}$ or $\phi_{c}$. The pair $(\phi, \phi)$ is a MPNE of the differential game (1)-(2). At any time $t>0$ the players only have access to the current value of the state. Given that the system is autonomous the players "forget" at $t>0$ any reference to the initial state of the system. That is, if at any time $t>0$ the game starting at time $t$ with an arbitrary initial state $y, \Gamma(t, y)$ in (7)-(8), is considered, then the pair $(\phi, \phi)$ is a MPNE of the differential game $\Gamma(t, y)$. Therefore, none of the players will be interested in unilaterally implementing a strategy different from $\phi$.

Remark 9 It is worth noting that, interestingly enough, in all cases the players implement a symmetric MPNE without imposing artificially symmetric play.

\section{Concluding remarks}

Multiple equilibria in dynamic games are a fundamental feature of infinite horizon models. This paper analyzes the problem of how to select an equilibrium in a differential game when there is an infinite number of equilibria. Other authors have suggested various selection criteria for choosing one among the many equilibria. In the applied dynamic games literature, the problem of the selection of equilibria has been avoided by considering pre-play negotiations or communications between the players. In this paper, as in Dockner and Long (1993), we consider that pre-play communications and cheap talk are equivalent devices. The selection of a specific equilibrium is determined in a pre-play phase where agents have cheap talks and agree on the selection of the pair of strategies. The main objective of this paper was to analyze which would be the equilibrium played without pre-play communications in a well-known class of differential games.

In particular, we analyzed the linear-quadratic differential game proposed by Dockner and Long (1993) to study a transboundary pollution problem. Existing studies have shown that there exists a continuum of nonlinear symmetric equilibria. In this paper, we show that when symmetry is not imposed there is also a continuum of nonlinear nonsymmetric equilibria. Furthermore, if the initial state (the initial pollution stock) is small enough the nonlinear strategies are not used as optimal strategies regardless of the existence of pre-play communications. We concluded that if the environmental problem is initially moderate in the presence of multiple equilibria and without cheap talk, then the equilibria are symmetric piecewise linear Markov perfect Nash equilibria. If the environmental problem is not initially serious then both countries have an incentive to choose an initial emission rate as great as possible. As a consequence, the implemented symmetric piecewise linear MPNE gives the lowest payoff for both countries with symmetric MPNE. For large values of the initial pollution stock, when the environmental problem is initially important, the 
strategy is the symmetric nonlinear MPNE that gives the closest asymptotic steady-state to the cooperative steady-state. This result is consistent with the findings in Dockner and Long (1993) and Rubio and Casino (2002).

It is worth mentioning that the techniques and results presented here are applicable not only to other linear-quadratic differential games, but to more general differential games.

Finally, we want to emphasize two points of our analysis. The first point concerns the possibility for players to play asymmetric strategies. The conclusion would be different if players were restricted to using symmetric strategies. The second point refers to the fact that although symmetric play has not been imposed, the equilibria actually implemented are symmetric.

\section{References}

1. Başar T (1977) Informationally nonunique equilibrium solutions in differential games. SIAM Journal on Control and Optimization 15(4): 636-660.

2. Benchekroun H, Long NV (2008) The build-up of cooperative behavior among noncooperative selfish agents. Journal of Economic Behavior \& Organization 67: 239-252.

3. Cartigny P, Michel P (2003) On the selection of one feedback Nash equilibrium in discounted linear-quadratic games. Journal of Optimization Theory and Applications 117(2): 231-243.

4. Chiarella C, Kemp MC, Long NV (1984) On the economics of international fisheries. International Economic Review 25: 85-92.

5. De Frutos J, Martín-Herrán G (2015) Does flexibility facilitate sustainability of cooperation over time? A case study from environmental economics. Journal of Optimization Theory and Applications 165: 657-677.

6. Dockner EJ, Jørgensen S, Long NV, Sorger G (2000) Differential games in economics and management science. Cambridge University Press, Cambridge.

7. Dockner EJ, Long NV (1993) International pollution control: cooperative versus noncooperative strategies. Journal of Environmental Economics and Management 24: 13-29.

8. Dockner EJ, Long NV, Sorger G (1996) Analysis of Nash equilibria in a class of capital accumulation games. Journal of Economic Dynamics and Control 20: 1209-1235.

9. Dockner E, Sorger G (1996) Existence and properties of equilibria for a dynamic game on productive assets. Journal of Economic Theory 71: 209-227.

10. Dockner E, Wagener F (2014) Markov perfect Nash equilibria in models with a single capital stock. Economic Theory 56: 585-625.

11. Driskill R (1997) Durable goods monopoly, increasing marginal cost and depreciation. Economica 64: 137-154.

12. Feichtinger G, Wirl F (1993) A dynamic variant of the battle of the sexes. International Journal of Game Theory 22: 359-380.

13. Fujiwara K (2008) Duopoly can be more anti-competitive than monopoly. Economics Letters 101: 217-219.

14. Fujiwara K (2009) Why environmentalists resist trade liberalization. Environmental and Resource Economics 44: 71-84.

15. Fujiwara K (2010) When are voluntary export restraints voluntary? A differential game approach. Australian Economic Papers, June: 101-110.

16. Fujiwara K, Matsueda N (2009) Dynamic voluntary provision of public goods: A generalization. Journal of Public Economic Theory 11(1): 27-36.

17. Kossioris G, Plexousakis M, Xepapadeas A, de Zeeuw A, Maler KG (2008) Feedback Nash equilibria for non-linear differential games in pollution control. Journal of Economic Dynamics and Control 32: 1312-1331.

18. Haurie A, Krawczyk JB, Zaccour G (2012) Games and Dynamic Games. World Scientific, Singapore. 
19. Ihori T, Itaya J (2001) A dynamic model of fiscal reconstruction. European Journal of Political Economy 17: 779-797.

20. Martín-Herrán G, Rincón-Zapatero JP (2002) Computation of Markov perfect Nash equilibria without Hamilton-Jacobi-Bellman equations. In G. Zaccour (ed.) Optimal Control and Differential Games. Essays in honor of Steffen Jørgensen. Kluwer, pp. 135-151.

21. Martín-Herrán G, Rincón-Zapatero JP (2005) Efficient Markov perfect Nash equilibria: Theory and applications to dynamic fishery games. Journal of Economic Dynamics \& Control 29: 1073-1096.

22. Piga CAG (2000) Competition in a duopoly with sticky price and advertising. International Journal of Industrial Organization 18: 595-614.

23. Rincón-Zapatero JP (2004) Characterization of Markovian equilibria in a class of differential games. Journal of Economic Dynamics \& Control 28: 1243-1266.

24. Rincón-Zapatero JP, Martín-Herrán G, Martínez J (2000) Identification of efficient subgame perfect Nash equilibria in a class of differential games. Journal of Optimization Theory and Applications 104: 235-242.

25. Rincón-Zapatero JP, Martínez J, Martín-Herrán G (1998) New method to characterize subgame perfect Nash equilibria in differential games. Journal of Optimization Theory and Applications 96: 377-395.

26. Rowat C (2007) Non-linear strategies in a linear quadratic differential game. Journal of Economic Dynamics \& Control 31: 3779-3202.

27. Rubio SJ, Casino B (2002) A note on cooperative versus non-cooperative strategies in international pollution control. Resource and Energy Economics 24: 251-261.

28. Rubio SJ, Casino B (2003) Strategic behavior and efficiency in the common property extraction of groundwater. Environmental and Resource Economics 26: 73-87.

29. Shibata A (2002) Strategic interactions in a growth model with infrastructure capital. Metroeconomica 53(4): 434-460.

30. Tasneem D, Engle-Warnick J, Benchekroun H (2017) An experimental study of a common property renewable resource game in continuous time. Journal of Economic Behavior \& Organization 140: 91-119.

31. Tsutsui S, Mino K (1990) Nonlinear Strategies in Dynamic Duopolistic Competition with Sticky Prices. Journal of Economic Theory 52: 136-161.

32. Wirl F (1994) Pigouvian taxation of energy for flow and stock externalities and strategic, noncompetitive energy pricing. Journal of Environmental Economics and Management 26: $1-18$.

33. Wirl F (1996) Dynamic voluntary provision of public goods: Extension to nonlinear strategies. European Journal of Political Economy 12: 555-560.

34. Wirl F (2007) Do multiple Nash equilibria in Markov strategies mitigate the tradegy of the commons?. Journal of Economic Dynamics \& Control 31: 3723-3740.

35. Wirl F, Dockner E (1995) Leviathan governments and carbon taxes: Costs and potential benefits. European Economic Review 39: 1215-1236.

36. Wirl F, Feichtinger G (2002) Intrafamiliar consumption and saving under altruism and wealth considerations. Economica 69: 93-111.

37. Zagonari F (1998) International pollution problems: Unilateral initiatives by environmental groups in one country. Journal of Environmental Economics and Management 36: 46-69. 